\title{
Boundary entropy of supersymmetric Janus solutions
}

\author{
Marco Chiodaroli, ${ }^{a}$ Michael Gutperle $^{a}$ and Ling-Yan Hung ${ }^{b}$ \\ ${ }^{a}$ Department of Physics and Astronomy, University of California, \\ Los Angeles, CA 90095, U.S.A. \\ ${ }^{b}$ Perimeter Institute, \\ Waterloo, Ontario N2L 2Y5, Canada \\ E-mail: mchiodar@ucla.edu, gutperle@physics.ucla.edu, \\ jhung@perimeterinstitute.ca
}

ABSTRACT: In this paper we compute the holographic boundary entropy for half-BPS Janus deformations of the $A d S_{3} \times S^{3} \times T^{4}$ vacuum of type IIB supergravity. Previous work [24] has shown that there are two independent deformations of this sort. In one case, the six-dimensional dilaton jumps across the interface, while the other case displays a jump of axion and four-form potential.

In case of a jump of the six-dimensional dilaton, it is possible to compare the holographic result with the weak-coupling result for a two-dimensional interface CFT where the radii of the compactified bosons jump across the interface. We find exact agreement between holographic and CFT results. This is to be contrasted with the holographic calculation for the non-supersymmetric Janus solution, which agrees with the CFT result only at the leading order in the jump parameter.

We also examine the implications of the holographic calculation in case of a solution with a jump in the axion, which can be associated with a deformation of the CFT by the $Z_{2}$-orbifold twist operator.

KeYwords: Gauge-gravity correspondence, AdS-CFT Correspondence, Field Theories in Lower Dimensions

ARXIV EPRINT: 1005.4433 


\section{Contents}

1 Introduction 1

2 Boundary and entanglement entropy 3

3 Ultraviolet regularization of AdS-sliced metrics 5

4 Non-supersymmetric Janus solution $\quad 7$

$\begin{array}{lll}4.1 & \text { Holographic boundary entropy } & 7\end{array}$

5 Supersymmetric Janus solution $\quad 9$

5.1 Calculation of the holographic boundary entropy 11

6 CFT calculation of the boundary entropy 13

$\begin{array}{lll}6.1 & \text { Supersymmetric boundary conditions } & 13\end{array}$

$\begin{array}{ll}\text { 6.2 Calculation of boundary entropy on the CFT side } & 15\end{array}$

$\begin{array}{lll}6.3 & \text { Boundary entropy for non-susy Janus } & 16\end{array}$

6.4 Boundary entropy for BPS Janus 16

$\begin{array}{ll}\text { 6.5 Deformation by the } Z_{2} \text { orbifold twist operator } & 17\end{array}$

$\begin{array}{lll}7 & \text { Conclusions } & 19\end{array}$

A BPS interface solution $\quad 20$

A.1 Local solutions 20

A.2 Page charges 21

A.3 BPS Janus solution 22

B Operator product of twist fields $\quad 22$

C Boundary entropy with both radii and $B$ field jumps 24

\section{Introduction}

Conformal field theories with boundaries or interfaces are the object of many interesting applications. A conformal boundary in a $d$-dimensional conformal field theory is a codimension one surface that is invariant under $d$-1-dimensional conformal transformations. In condensed matter physics, these theories are used to describe impurities in critical systems. Cardy [1] initiated the project of classifying all conformal boundary conditions for two-dimensional CFTs. In string theory, boundary conformal field theories are employed for the world-sheet description of D-branes. 
Conformal interfaces provide a generalization of boundary CFTs. In an interface theory, two different conformal theories, $C F T_{1}$ and $C F T_{2}$, are separated by a hypersurface of co-dimension one. The folding trick $[2,3]$ relates a two-dimensional conformal interface theory to a boundary CFT in the tensor product $C F T_{1} \otimes C F T_{2}$. Consequently, the folding trick can be employed to classify possible interface theories, calculate reflection and transmission coefficients for bulk excitations, study bulk and boundary perturbations and renormalization group flows.

In this paper, we will examine the boundary entropy, which can be obtained from the ground state degeneracy, or g-factor, of the boundary CFT [4]. The boundary entropy of the folded theory can be interpreted as the entropy associated with the interface. This quantity is universal and constitutes the analogue of the central charge for a boundary CFT. The boundary entropy can help classify conformal boundary conditions and give information about the low energy spectrum of the system. In fact, this quantity is measurable in experiments, as demonstrated by studies in candidate materials exhibiting the Kondoeffect [5]. In [6] it was further argued that the boundary entropy is related to the finite part of the entanglement entropy.

The AdS/CFT correspondence [7-9] is a powerful tool for studying conformal field theories employing dual gravitational theories in Anti de-Sitter spacetimes. In particular, the construction of defects and interfaces in the probe approximation was initiated in $[3,10,11]$, with the analysis of branes spanning $A d S_{d}$ submanifolds inside an $A d S_{d+1}$ space.

Moreover, the so-called Janus-solution was constructed in [12]. This solution is a fully back-reacted solution of type IIB supergravity that is locally asymptotic to $A d S_{5} \times S^{5}$. The Janus solution is the holographic dual of an interface theory in which the gauge coupling is constant throughout the bulk of two 3+1-dimensional half-spaces, but is allowed to jump across a planar 2+1-dimensional interface, where the half-spaces are glued together.

The literature examines many generalizations of the original Janus solution [13-23], including solutions where the interface preserves up to one half of the supersymmetries of the bulk theory.

In the present paper, we study supersymmetric solutions of type IIB supergravity which are locally asymptotic to $A d S_{3} \times S^{3} \times T^{4}$. These solutions were first constructed in $[24],{ }^{1}$ and are the holographic duals of various marginal deformations of two-dimensional $\mathcal{N}=(4,4)$ super-conformal field theory.

A prescription for the holographic calculation of the entanglement entropy of a conformal field theory was given in [28, 29]. In [30] this holographic prescription was used to calculate boundary entropy for probe branes and for a non-supersymmetric Janus solution with $A d S_{3} \times S^{3}$ asymptotics.

The goal of the present paper is to calculate the boundary entropy holographically for the half-BPS Janus solution of [24] and compare the result with a weak-coupling calculation in the dual two-dimensional CFT. In particular, we specialize to the case where only D1and $D 5$-brane charges are present. In this case, the Janus solution describes an interface where two operators in the CFT corresponding to the six dimensional dilaton and to the linear combination of the axion and RR four form.

\footnotetext{
${ }^{1}$ For earlier work in this direction see [25-27].
} 
The main result of this paper is the exact agreement between the two calculations in the case in which the interface CFT displays only a jump of the $T^{4}$ volume across the interface. This is in contrast to the non-supersymmetric case where the calculations only agree to the leading order in the deformation parameter. While we have not performed a rigorous calculation in the presence of $Z_{2}$ orbifold twist operator deformation jump on the CFT side, we have a number of observations, as explained in section 6 , that suggest complete agreement with the supergravity result also in this case.

The organization of the paper is as follows. In section 2, we briefly review the boundary entropy and its relation to the entanglement entropy. In addition, we give a brief review of the prescription for the holographic calculation of the entanglement entropy discussed in $[28,29]$. In section 3 , we discuss the regularization near the boundary of the $A d S_{3}$ space which will be important for the calculation of the boundary entropy. In section 4 , we review the non-supersymmetric $A d S_{3}$ Janus solution found in [31] and the holographic calculation of the interface entropy performed in [30]. We pay particular attention to the regularization of the holographic entropy function. In section 5 , we generalize the framework for the holographic computation of the entanglement entropy introduced in [28, 29] to the case of a spacetime with geometry of the form $A d S_{p} \times S^{q} \times \Sigma$, where $\Sigma$ is a two-dimensional surface. We examine the supersymmetric Janus solution obtained in [24], and compute the holographic entanglement entropy as a function of the deformation parameters. In section 6, we perform the CFT calculation of the boundary entropy in case of a jump in the radii of the compact bosons and find exact agreement with the result from section 5 . Moreover, we explore the implications of our result on the properties of correlators of $Z_{2}$ twist fields. We conclude the paper with a discussion of our results.

\section{Boundary and entanglement entropy}

The logarithm of the partition function for a two-dimensional conformal theory defined on a spatial segment of length $L$ is given by

$$
\log Z \sim \log \operatorname{tr}\left(e^{-\beta H_{A B}}\right)=\log \left(g_{A} g_{B}\right)+\frac{c \pi}{6 \beta} L
$$

This expression is valid in the limit $L \gg \beta$. Here $H_{A B}$ is the open string Hamiltonian associated with the conformal boundary conditions on the two ends of the strip, denoted by $A$ and $B$. The universal factor $g_{A}$ is interpreted as the ground state degeneracy, or g-factor, associated with the conformal boundary $A$ [4]. A modular transformation relates the open string annulus partition function to a closed string cylinder partition function,

$$
Z=\left\langle A\left|e^{-L H_{c l}}\right| B\right\rangle
$$

where $|A\rangle$ is the boundary state which realizes the boundary condition $A$ on the closed string channel CFT. In the limit $L / \beta \rightarrow \infty$, it can be shown that the $g_{A}$ factor is given by

$$
g_{A}=\langle 0 \mid A\rangle
$$

The logarithm of $g_{A}$ is called the boundary entropy and counts the ground state degeneracy of the boundary theory. 


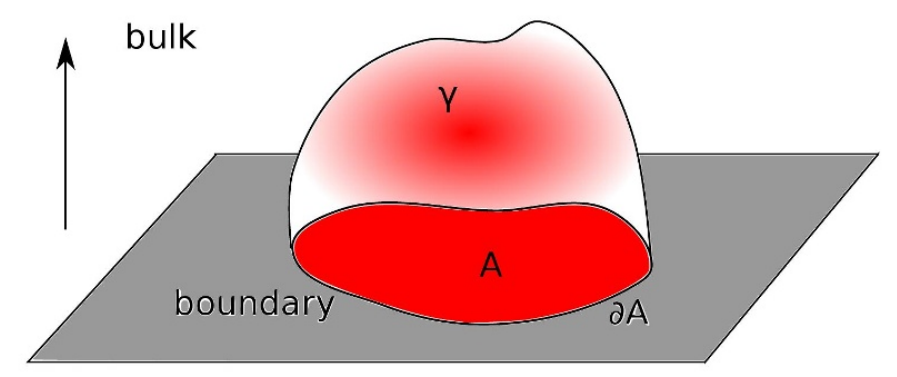

Figure 1. Minimal surface for the holographic calculation of boundary entropy.

It has been argued in [6] (see also [30] for a recent discussion) that the boundary entropy can be associated with the entanglement entropy in a system with a boundary. Consider a CFT defined on a half-line with a conformal boundary condition $A$ at $x=0$. The system can be divided into a subsystem $\mathcal{A}$, defined on the interval $[0, l]$, and its complement $\mathcal{B}$, defined on $[l, \infty]$. The total space of states is given by the product $H=H_{\mathcal{A}} \otimes H_{\mathcal{B}}$. A reduced density matrix can be defined by tracing over all states in $\mathcal{B}$,

$$
\rho_{\mathcal{A}}=\operatorname{tr}_{H_{\mathcal{B}}} \rho
$$

where $\rho$ is the density matrix of the total system (at zero temperature this is just the projector on the ground state). The entanglement entropy is then defined as

$$
S_{\mathcal{A}}=-\operatorname{tr}_{H_{\mathcal{A}}} \rho_{\mathcal{A}} \log \rho_{\mathcal{A}}
$$

The entanglement entropy takes the following form in the limit of large $l[6]$,

$$
S_{\mathcal{A}}=\frac{c}{6} \log \frac{l}{a}+\log g_{A}
$$

where $a$ is a UV cutoff and $g_{A}$ is precisely the g-factor (2.3) associated with the conformal boundary $A .^{2}$

A proposal to calculate the entanglement entropy of a $C F T_{d}$ with a dual description as a gravitational theory in $A d S_{d+1}$ was discussed in [28, 29]. Working in Poincaré coordinates, the CFT is defined on Minkowski space $R^{1, d-1}$ which can be thought of as the boundary of $A d S_{d+1}$. We consider a static setup where we choose a particular time slice on the boundary.

The subsystem $\mathcal{A}$ is a $d$-dimensional spatial region in the constant-time slice. The boundary of $\mathcal{A}$ will be denoted by $\partial \mathcal{A}$ (see figure 1). One can find a static minimal surface $\gamma_{\mathcal{A}}$ which extends into the $A d S_{d+1}$ bulk and ends on $\partial \mathcal{A}$ as one approaches the boundary of $A d S_{d+1}$. The holographic entanglement entropy can then be calculated as follows [28, 29],

$$
S_{A}=\frac{\operatorname{Area}\left(\gamma_{\mathcal{A}}\right)}{4 G_{N}^{(d+1)}}
$$

\footnotetext{
${ }^{2}$ An additional term $c^{\prime}$ was included in [6]. This term is non-universal and independent of the presence of the boundary. We compute $g_{A}$ as the difference between the entanglement entropy with an interface and the entropy of the same system without an interface. Therefore, $c^{\prime}$ will not contribute to our results.
} 
where Area $\left(\gamma_{\mathcal{A}}\right)$ denotes the area of the minimal surface $\gamma_{\mathcal{A}}$ and $G_{N}^{(d+1)}$ is the Newton constant for $A d S_{d+1}$ gravity. In the case of $A d S_{3}$, the area $\mathcal{A}$ is an interval and the boundary $\partial \mathcal{A}$ is a collection of points. The minimal surface is a spacelike geodesic connecting these points.

\section{Ultraviolet regularization of AdS-sliced metrics}

The starting point for the construction of the relevant Janus solutions is the $A d S_{2}$ slicing of $A d S_{3}$, given by

$$
d s^{2}=R_{\mathrm{AdS}_{3}}^{2}\left(d x^{2}+\cosh ^{2} x \frac{d z^{2}-d t^{2}}{z^{2}}\right)
$$

The structure of the $A d S_{3}$ boundary in this coordinates is more complicated than the one encountered with the Poincaré patch. In particular, there are three boundary components. Two $1+1$-dimensional half-spaces can be reached by taking the limit $x \rightarrow \pm \infty$. The two half spaces are glued together at a $0+1$-dimensional world-line, which is reached by taking $z \rightarrow 0$. It is useful to perform the change of coordinates,

$$
\zeta=\tanh (x)
$$

Under this change, the metric (3.1) becomes

$$
d s^{2}=R_{\mathrm{AdS}_{3}}^{2}\left(\frac{d \zeta^{2}}{\left(1-\zeta^{2}\right)^{2}}+\frac{1}{1-\zeta^{2}} \frac{d z^{2}-d t^{2}}{z^{2}}\right)
$$

In the calculation of the entanglement entropy one obtains expressions which diverge near the boundary of the bulk spacetime. In the field theory, this phenomenon can be interpreted as an ultraviolet divergence. Hence, in order to perform the calculation we have to introduce an ultraviolet regulator. The introduction of Fefferman-Graham coordinates provides a systematic procedure for regularizing the AdS-sliced metrics, as discussed in appendix B of [33].

In case of the metric (3.1), the Fefferman-Graham coordinates are equivalent to the Poincaré patch, and can be introduced as

$$
\zeta=\frac{\eta}{\sqrt{\xi^{2}+\eta^{2}}}, \quad z=\sqrt{\xi^{2}+\eta^{2}}
$$

With this change, the metric becomes

$$
d s^{2}=\frac{R_{\mathrm{AdS}_{3}}^{2}}{\xi^{2}}\left(d \xi^{2}+d \eta^{2}-d t^{2}\right)
$$

The boundary of the bulk spacetime is now reached by $\xi \rightarrow 0$. The interface is located at $\xi=0, \eta=0$. Note that there are subtle issues with the order of limits if one approaches the boundary and the interface at the same time.

The global coordinate change to a Fefferman-Graham system is not known for the supersymmetric Janus solution employed in this paper. In the following analysis, we will be interested mainly in imposing an ultraviolet cutoff in the region away from the interface, 
i.e. where $z \gg 0$. Note that a more complete analysis is needed for holographic calculations of bulk/boundary operator expansions in the CFT, as one is interested in the behavior of the solution as $z \rightarrow 0$.

By taking $x \rightarrow \pm \infty$ and keeping $z$ finite, one reaches the boundary staying away from the interface. In this limit, we will encounter metrics with a slightly more general form than (3.1),

$$
\lim _{x \rightarrow \pm \infty} d s^{2}=R_{\mathrm{AdS}_{3}}^{2}\left(d x^{2}+\frac{\lambda_{ \pm}}{4} e^{ \pm 2 x} \frac{d z^{2}-d t^{2}}{z^{2}}\right)+o(1)
$$

One can absorb the constant $\lambda_{ \pm}$by a shift in $x$,

$$
x=\tilde{x} \mp \frac{1}{2} \log \left(\lambda_{ \pm}\right)
$$

and one gets

$$
\lim _{x \rightarrow \pm \infty} d s^{2}=R_{\mathrm{AdS}_{3}}^{2}\left(d \tilde{x}^{2}+\frac{1}{4} e^{ \pm 2 \tilde{x}} \frac{d z^{2}-d t^{2}}{z^{2}}\right)+o(1)
$$

In this limit, the Fefferman-Graham coordinate change becomes

$$
\begin{aligned}
& \tilde{x} \rightarrow+\infty, \quad \xi \rightarrow 0, \quad \eta>0: \quad e^{-2 \tilde{x}}=\frac{1}{4} \frac{\xi^{2}}{\eta^{2}}, \quad z=\eta\left(1+\frac{1}{2} \frac{\xi^{2}}{\eta^{2}}\right) \\
& \tilde{x} \rightarrow-\infty, \quad \xi \rightarrow 0, \quad \eta<0: \quad e^{2 \tilde{x}}=\frac{1}{4} \frac{\xi^{2}}{\eta^{2}}, \quad z=|\eta|\left(1+\frac{1}{2} \frac{\xi^{2}}{\eta^{2}}\right)
\end{aligned}
$$

The above equations were obtained using the relations

$$
\begin{array}{ll}
\tilde{x} \rightarrow+\infty, & \zeta=1-2 e^{-2 \tilde{x}}+\cdots \\
\tilde{x} \rightarrow-\infty, & \zeta=-1+2 e^{2 \tilde{x}}+\cdots
\end{array}
$$

In both cases, the metric becomes

$$
\lim _{|x| \rightarrow \pm \infty} d s^{2}=\frac{R_{\mathrm{AdS}_{3}}^{2}}{\xi^{2}}\left(d \xi^{2}+d \eta^{2}-d t^{2}\right)+\cdots
$$

The interface is located at $\eta=0$, and in the two cases the coordinate $\eta$ is restricted to $\eta>0$ and $\eta<0$ respectively. Note that near $\eta=0$ the change of coordinates is more complicated. In particular, the coordinate change (3.9) appears not to be smooth at $\eta=0$. However, this change of coordinates is valid as long as one is not approaching the interface while approaching the boundary. If we now set the cutoff at $\xi=\epsilon$ and consider a point $|\eta|=z_{0}$, we can use (3.7) and (3.9) to obtain a relation between the cutoff $\epsilon$ and $x$ in the two asymptotic regions,

$$
\begin{array}{ll}
x \rightarrow+\infty: & \epsilon=\frac{2 z_{0}}{\sqrt{\lambda_{+}}} e^{-x_{\infty}} \\
x \rightarrow-\infty: & \epsilon=\frac{2 z_{0}}{\sqrt{\lambda_{-}}} e^{x_{-\infty}}
\end{array}
$$




\section{Non-supersymmetric Janus solution}

The boundary entropy for a non-supersymmetric Janus solution with $A d S_{3}$ asymptotics was calculated in [30]. We reproduce the result of [30] by introducing an ultraviolet regulator near the $A d S_{3}$ boundary.

The non-BPS Janus deformation of the $A d S_{3} \times S^{3} \times T^{4}$ vacuum of type IIB supergravity was found in [31]. The ten-dimensional metric is given by

$$
d s^{2}=e^{\frac{\phi}{2}}\left(d s_{3}^{2}+d s_{\mathrm{S}^{3}}^{2}\right)+e^{-\phi / 2} d s_{\mathrm{M}_{4}}^{2}
$$

where the three-dimensional metric $d s_{3}^{2}$ is

$$
d s_{3}^{2}=R_{\mathrm{AdS}_{3}}^{2}\left(d x^{2}+f(x) d s_{\mathrm{AdS}_{2}}^{2}\right)
$$

the function $f(x)$ has the following expression,

$$
f(x)=\frac{1}{2}\left(1+\sqrt{1-2 \gamma^{2}} \cosh (2 x)\right)
$$

The dilaton is given by

$$
\phi(x)=\phi_{0}+\frac{1}{\sqrt{2}} \log \left(\frac{1+\sqrt{1-2 \gamma^{2}}+\sqrt{2} \gamma \tanh x}{1+\sqrt{1-2 \gamma^{2}}-\sqrt{2} \gamma \tanh x}\right)
$$

Note that if we consider the compactification of the ten-dimensional theory to sixdimensions, the resulting action is not in the Einstein frame. To bring the six-dimensional action to the Einstein frame we have to multiply the six dimensional part of the metric by $e^{-\phi / 2}$,

$$
d s_{6, E}^{2}=R_{\mathrm{AdS}_{3}}^{2}\left(d x^{2}+f(x) d s_{\mathrm{AdS}_{2}}^{2}\right)+R_{\mathrm{AdS}_{3}}^{2}\left(d y^{2}+\sin ^{2} y d s_{\mathrm{S}^{2}}^{2}\right)
$$

The expansion of $f(x)$ as $x \rightarrow \pm \infty$ is

$$
\lim _{x \rightarrow \pm \infty} f(x)=\frac{1}{4} \sqrt{1-2 \gamma^{2}} e^{ \pm 2 x}+o(1)
$$

Comparison with (3.6) shows that in this case the constants $\lambda_{ \pm}$are given by

$$
\lambda_{ \pm}=\sqrt{1-2 \gamma^{2}}
$$

\subsection{Holographic boundary entropy}

The geodesic which was used in [30] to compute the entanglement entropy has a particularly simple form: the $z$ coordinate stays constant,

$$
z=z_{0}
$$

while $x$ varies from $-\infty$ to $+\infty$. This corresponds to a symmetric region around the interface as depicted in figure 2 . 


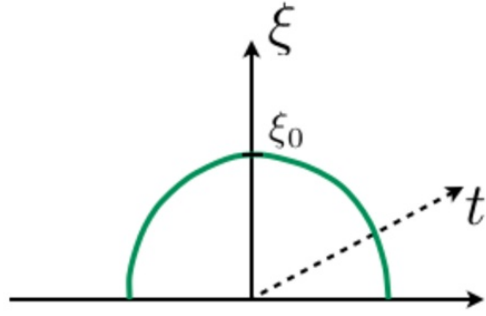

(a)

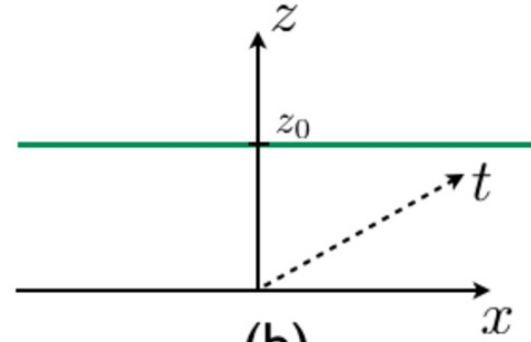

(b)

Figure 2. (a) Minimal surface for the holographic entanglement entropy in Poincare coordinates (b) Minimal surface in the $A d S_{2}$ slicing of $A d S_{3}$.

With this choice, the subsystem $\mathcal{A}$ is a symmetric interval around the interface given by $\left[-z_{0}, z_{0}\right]$. In this particular case, the geodesic length is given by

$$
\Gamma(\gamma)=R_{\mathrm{AdS}_{3}} \int d x=R_{\mathrm{AdS}_{3}}\left(x_{\infty}(\gamma)-x_{-\infty}(\gamma)\right)
$$

where $x_{ \pm \infty}$ is the $x$ coordinate evaluated at the cutoff. We can now use the relation between $x_{ \pm \infty}$ and the cutoff $\epsilon$,

$$
\begin{aligned}
x_{\infty} & =-\log \epsilon-\frac{1}{2} \log \lambda_{+}+\log \left(2 z_{0}\right) \\
x_{-\infty} & =\log \epsilon+\frac{1}{2} \log \lambda_{-}-\log \left(2 z_{0}\right)
\end{aligned}
$$

Hence

$$
\begin{aligned}
\Gamma(\gamma) / R_{\mathrm{AdS}_{3}} & =x_{\infty}(\gamma)-x_{-\infty}(\gamma) \\
& =-2 \log \epsilon-\frac{1}{2}\left(\log \lambda_{+}+\log \lambda_{-}\right)+2 \log \left(2 z_{0}\right) \\
& =-2 \log \epsilon-\log \left(\sqrt{1-2 \gamma^{2}}\right)+2 \log \left(2 z_{0}\right)
\end{aligned}
$$

The entanglement entropy is then given by the difference between the geodesic length in the Janus geometry and the length in $A d S_{3}$ evaluated at the same value of the cutoff $\epsilon$. The difference of the two lengths is then finite and independent of the cutoff,

$$
S_{\mathrm{bdy}}=\frac{\Gamma(\gamma)-\Gamma(0)}{G_{3}}=-\frac{R_{\mathrm{AdS}_{3}}}{4 G_{3}} \log \left(\sqrt{1-2 \gamma^{2}}\right)=N_{\mathrm{D} 1} N_{\mathrm{D} 5} \log \frac{1}{\sqrt{1-2 \gamma^{2}}}
$$

We used the Brown-Henneaux formula [34] for the central charge of the the CFT,

$$
\frac{3 R_{\mathrm{AdS}_{3}}}{2 G_{3}}=c=6 N_{\mathrm{D} 1} N_{\mathrm{D} 5}
$$

$N_{\text {D1/D5 }}$ are the number of $D 1$ - and $D 5$-branes which realize the CFT. With equation (4.12) we have reproduced the result for the boundary entropy (4.12) found in [30]. 


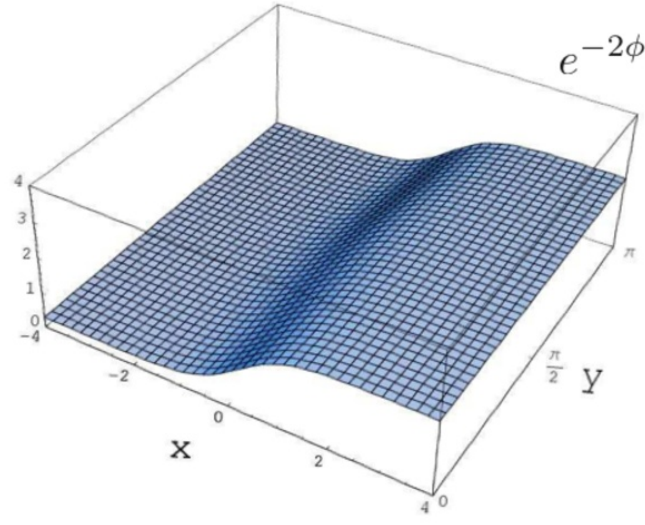

(a)

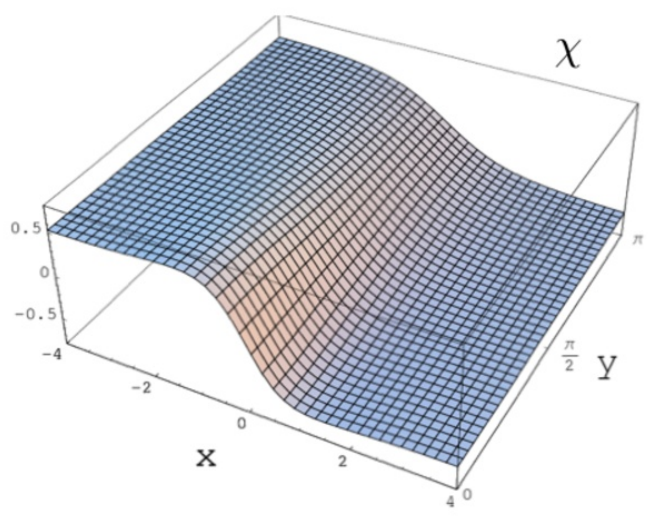

(b)

Figure 3. (a) Plot of the dilaton $e^{-2 \phi}$ for the deformation $\theta=0, \psi=\frac{1}{2}$ depicting the jump in the dilaton as $x \rightarrow \pm \infty$. (b) Plot of the axion $\chi$ for the solution $\psi=0, \theta=\frac{1}{2}$ depicting the jump in the axion as $x \rightarrow \pm \infty$.

\section{Supersymmetric Janus solution}

In this section we will calculate the holographic boundary entropy for the supersymmetric Janus solution found in [24]. More details about the solution can be found in appendix A.

We consider type IIB supergravity compactified on $M_{4}$, where $M_{4}$ is either $K_{3}$ or $T^{4}$. In the present paper we will use a four-torus since the dual CFT is simpler.

The ten-dimensional metric ansatz is a fibration of $A d S_{2} \times S^{2} \times M_{4}$ over a two dimensional Riemann surface $\Sigma$

$$
d s^{2}=f_{1}^{2} d s_{\mathrm{AdS}_{2}}^{2}+f_{2}^{2} d s_{\mathrm{S}^{2}}^{2}+f_{3}^{2} d s_{\mathrm{M}_{4}}^{2}+\rho^{2} d z d \bar{z}
$$

All fields will depend on the coordinates $z, \bar{z}$ of $\Sigma$. For the supersymmetric Janus solution, $\Sigma$ is an infinite strip with coordinates

$$
w=x+i y, \quad x \in[-\infty,+\infty], y \in[0, \pi]
$$

The boundaries of the strip are at $y=0, \pi$. The supersymmetric Janus solution depends on four parameters $k, L, \theta$ and $\psi$. $\psi$ parameterizes the jump of the six-dimensional dilaton across the interface and $\theta$ parameterizes the jump of the axion. ${ }^{3}$ Note that setting $\psi=0$ and $\theta=0$ gives the $A d S_{3} \times S^{3}$ vacuum. The dilaton and axion are given by (see figure 3 )

$$
\begin{aligned}
e^{-2 \phi} & =k^{4} \frac{\cosh ^{2}(x+\psi) \operatorname{sech}^{2} \psi+\left(\cosh ^{2} \theta-\operatorname{sech}^{2} \psi\right) \sin ^{2} y}{(\cosh x-\cos y \tanh \theta)^{2}} \\
\chi & =-\frac{k^{2}}{2} \frac{\sinh 2 \theta \sinh x-2 \tanh \psi \cos y}{\cosh x \cosh \theta-\cos y \sinh \theta}
\end{aligned}
$$

\footnotetext{
${ }^{3} \mathrm{~A}$ constant $c$ which appeared in [24] has been set to one. Moreover, in the previous paper we used the scalar $\Phi$ which is related to the standard dilaton by $\phi=-2 \Phi$.
} 
The metric factors for $\Sigma$ and $T^{4}$ are given by

$$
\begin{aligned}
\rho^{4} & =e^{-\phi} \frac{L^{2}}{k^{2}} \frac{\cosh ^{2} x \cosh ^{2} \theta-\cos ^{2} y \sinh ^{2} \theta}{\cosh ^{2}(x+\psi)} \cosh ^{4} \psi \\
f_{3}^{4} & =e^{-\phi} \frac{4}{k^{2}} \frac{\cosh x \cosh \theta-\cos y \sinh \theta}{\cosh x \cosh \theta+\cos y \sinh \theta}
\end{aligned}
$$

The following expressions for the $A d S_{2}$ and $S^{2}$ metric factors will be useful,

$$
\begin{aligned}
& \frac{f_{1}^{2}}{\rho^{2}}=\frac{\cosh ^{2}(x+\psi)}{\cosh ^{2} \theta \cosh ^{2} \psi} \\
& \frac{\rho^{2}}{f_{2}^{2}}=\frac{1}{\sin ^{2} y}+\frac{\cosh ^{2} \theta \cosh ^{2} \psi-1}{\cosh ^{2}(x+\psi)}
\end{aligned}
$$

The Page charges, defined in appendix A, can be calculated for the present solution. One finds that the fundamental string and $N S 5$-brane charges vanish, i.e. $Q_{\mathrm{F} 1}=0$ and $Q_{\mathrm{NS} 5}=$ 0 . The $D 1$ - and $D 5$-brane charges are given by

$$
\begin{aligned}
Q_{\mathrm{D} 5} & =4 \pi^{2} k L \cosh \psi \cosh \theta \\
Q_{\mathrm{D} 1} & =\frac{16 \pi^{2} L}{k} \cosh \psi \cosh \theta
\end{aligned}
$$

The dual CFT is a $\mathcal{N}=(4,4)$ superconformal field theory which can be understood, at a particular point of its moduli space, as the $\left(M^{4}\right)^{Q_{\mathrm{D} 1} Q_{\mathrm{D} 5}} / S_{Q_{\mathrm{D} 1} Q_{\mathrm{D} 5}}$ orbifold sigma-model. The central charge of the CFT can be expressed in terms of the charges as follows

$$
c=\frac{6}{4 \pi k_{10}^{2}} Q_{\mathrm{D} 1} Q_{\mathrm{D} 5}=\frac{3 \times 32 \pi^{3} L^{2}}{k_{10}^{2}} \cosh ^{2} \psi \cosh ^{2} \theta
$$

The limit $x \rightarrow \pm \infty$ corresponds to approaching the AdS boundary, where the scalar fields behave as follows

$$
\lim _{x \rightarrow \pm \infty} e^{-\phi} f_{3}^{4}=4 k^{2} \frac{e^{\mp 2 \psi}}{\cosh ^{2} \psi}
$$

This combination of scalars is precisely the six-dimensional dilaton, and is dual to the volume of the four-torus of the orbifold CFT. Hence, the deformation corresponds to an interface CFT where the volume jumps across the interface.

Similarly, the combination

$$
\lim _{x \rightarrow \pm \infty} e^{\frac{\phi}{2}} f_{3}^{2} \chi-4 \frac{e^{-\frac{\phi}{2}}}{f_{3}^{2}} C_{4}= \pm 4 k \sinh \theta
$$

is dual to the $Z_{2}$ orbifold twist operator of the $\left(M_{4}\right)^{N} / S_{N}$ orbifold. Solutions with nonzero $\theta$ correspond to interfaces in which the orbifold CFTs on each side are at two different points in their moduli space. 


\subsection{Calculation of the holographic boundary entropy}

The calculation of the holographic boundary entropy for the non-supersymmetric Janus solution was simplified by the fact the three-sphere was left undeformed. The calculation of the minimal surface could be done by compactifying the ten-dimensional theory on $S^{3}$ and $T^{4}$ obtaining an effective three-dimensional theory. This reduction is equivalent to considering a minimal surface wrapping these spaces, in accordance with the principle that the entanglement entropy is a trace over all states.

Supersymmetric Janus solution in various dimensions have a more complicated structure. In general, their geometry is given by an $A d S_{p} \times S^{q}$ fibration over a Riemann surface $\Sigma$. In analogy with the simpler case of non-supersymmetric solutions, we propose to compute the entanglement entropy using a $p+q$-dimensional minimal surface which spans the sphere $S^{q}$ as well as the Riemann surface $\Sigma$.

It is useful to consider an intermediate step and compactify the metric (5.1) along the compact directions. In order to have the correct Einstein-Hilbert action, we rescale by a factor of $f_{3}^{-2}$ to obtain the resulting metric in the six-dimensional Einstein frame,

$$
d s_{6, E}^{2}=\rho^{2} f_{3}^{2}\left(\frac{\cosh ^{2}(x+\psi)}{\cosh ^{2} \psi \cosh ^{2} \theta} \frac{d z^{2}-d t^{2}}{z^{2}}+d x^{2}+d y^{2}\right)+f_{2}^{2} f_{3}^{2} d s_{\mathrm{S}^{2}}^{2}
$$

We now use the following expressions for the limit $x \rightarrow \pm \infty$,

$$
\begin{aligned}
\lim _{x \rightarrow \pm \infty} e^{-\phi} & =k^{2} e^{ \pm \psi} \operatorname{sech} \psi \\
\lim _{x \rightarrow \pm \infty} \rho^{2} & =L e^{\mp \psi / 2} \cosh \theta \cosh ^{3 / 2} \psi \\
\lim _{x \rightarrow \pm \infty} f_{3}^{2} & =2 \frac{e^{ \pm \psi / 2}}{\cosh ^{1 / 2} \psi} \\
\lim _{x \rightarrow \pm \infty} f_{2}^{2} & =\rho^{2} \sin ^{2} y=L e^{\mp \psi / 2} \cosh \theta \cosh ^{3 / 2} \psi \sin ^{2} y
\end{aligned}
$$

Hence the metric (5.11) becomes

$$
\lim _{x \rightarrow \pm \infty} d s_{6, E}^{2}=2 L \cosh \theta \cosh \psi\left(\frac{e^{ \pm 2 \psi}}{\cosh ^{2} \psi \cosh ^{2} \theta} \frac{e^{ \pm 2 x}}{4} \frac{d z^{2}-d t^{2}}{z^{2}}+d x^{2}+d y^{2}+\sin ^{2} y d s_{\mathrm{S}_{2}}^{2}\right)
$$

We can then identify

$$
R_{\mathrm{AdS}_{3}}^{2}=2 L \cosh \theta \cosh \psi
$$

Note that the AdS radius is the same on both sides of the interface. The constants $\lambda_{ \pm}$are then given by

$$
\lambda_{ \pm}=\frac{e^{ \pm 2 \psi}}{\cosh ^{2} \psi \cosh ^{2} \theta}
$$

This expression gives us the relevant formula for the cutoff. We consider the fourdimensional minimal surface located at $z=z_{0}, t=t_{0}$ and spanning $x, y$ and the $S_{2}$ 
directions. Using the six-dimensional metric (5.11) we obtain

$$
\begin{aligned}
A(\theta, \psi) & =\int d \Omega_{2} \int d x \int d y\left(f_{2}^{2} f_{3}^{2}\right) \times\left(\rho^{2} f_{3}^{2}\right) \\
& =V_{\mathrm{S}^{2}} \int d x d y \frac{H^{2} \rho^{2}}{f_{1}^{2}} \\
& =4 L^{2} \cosh ^{2} \psi \cosh ^{2} \theta V_{\mathrm{S}^{2}} \int d y \sin ^{2} y \int d x \\
& =4 L^{2} \cosh ^{2} \psi \cosh ^{2} \theta V_{\mathrm{S}^{3}} \int d x \\
& =R_{\mathrm{AdS}_{3}}^{4} V_{\mathrm{S}^{3}} \int d x
\end{aligned}
$$

We need to compare this area to the one of a minimal surface in an $A d S_{3} \times S^{3}$ space with the same curvature radius. Instead of (5.11), we have the metric

$$
d s_{\mathrm{AdS}_{3} \times \mathrm{S}^{3}}^{2}=R_{\mathrm{AdS}_{3}}^{2}\left(\frac{e^{ \pm 2 x}}{4} \frac{d z^{2}-d t^{2}}{z^{2}}+d x^{2}+d y^{2}+\sin ^{2} y d s_{\mathrm{S}_{2}}^{2}\right)
$$

which is the $A d S_{3} \times S^{3}$ metric with the same cosmological constant as (5.11). The area in $A d S_{3} \times S^{3}$ is

$$
A_{0}=R_{\mathrm{AdS}_{3}}^{4} \int d \Omega_{2} \int d x \int d y \sin ^{2} y=R_{\mathrm{AdS}_{3}}^{4} V_{\mathrm{S}_{3}} \int d x
$$

and the difference is

$$
A(\theta, \psi)-A_{0}=R_{\mathrm{AdS}_{3}}^{4} V_{\mathrm{S}^{3}}(\Gamma(\theta, \psi)-\Gamma(0))
$$

where the geodesic length $\Gamma$ was defined in (4.9). We can now apply the cutoff prescription discussed in section 3 and get

$$
A(\theta, \psi)-A_{0}=-R_{\mathrm{AdS}_{3}}^{4} V_{\mathrm{S}^{3}} \frac{1}{2}\left(\log \lambda_{+}+\log \lambda_{-}\right)=2 R_{\mathrm{AdS}_{3}}^{4} V_{\mathrm{S}^{3}} \log (\cosh \theta \cosh \psi)
$$

giving the boundary entropy

$$
\begin{aligned}
S_{\mathrm{bdy}} & =\frac{A(\theta, \psi)-A_{0}}{4 G_{6}} \\
& =\frac{16 \pi^{2} L^{2}}{4 G_{6}} \cosh ^{2} \psi \cosh ^{2} \theta \log (\cosh \theta \cosh \psi) \\
& =\frac{32 \pi^{3} L^{2}}{k_{10}^{2}} \cosh ^{2} \psi \cosh ^{2} \theta \log (\cosh \theta \cosh \psi)
\end{aligned}
$$

where we used the relation between the Newton's constant and $k_{10}$,

$$
\frac{1}{16 \pi G_{N}}=\frac{1}{2 k_{10}^{2}}
$$

In particular, setting $\theta=0$ gives

$$
S_{\text {bdy }}=\frac{32 \pi^{3} L^{2}}{k_{10}^{2}} \cosh ^{2} \psi \log (\cosh \psi)
$$

This expression gives the boundary entropy for a Janus solution in which only the sixdimensional dilaton jumps across the interface. 


\section{CFT calculation of the boundary entropy}

In this section we will discuss the calculation of the boundary entropy on the CFT side. Preservation of one half of the supersymmetries imposes specific boundary conditions on the fields at the interface. We use results in the literature to calculate the boundary entropy for the case where only the coupling constant jumps across the interface.

\subsection{Supersymmetric boundary conditions}

We now discuss the supersymmetries which are preserved in the presence of an interface in two-dimensional $\mathcal{N}=(4,4)$ super conformal field theory. For simplicity, we will focus on the free field limit, where the target space is simply the orbifold $\left(T^{4}\right)^{N} / S_{N}$. The free field action of the theory is given in [40], and can be written as follows in terms of the complex fields defined there,

$$
\begin{aligned}
S= & \frac{1}{2} \int d \tau d \sigma \frac{1}{2}\left(\partial_{+} X^{I} \partial_{-} X^{I^{\dagger}}+\partial_{-} X^{I} \partial_{+} X^{I^{\dagger}}\right) \\
& -\left(\Psi^{I} \partial_{-} \Psi^{I^{\dagger}}-\frac{1}{2} \partial_{-}\left(\Psi^{I} \Psi^{I^{\dagger}}\right)+\tilde{\Psi}^{I} \partial_{+} \tilde{\Psi}^{I \dagger}-\frac{1}{2} \partial_{+}\left(\tilde{\Psi}^{I} \tilde{\Psi}^{I \dagger}\right)\right)
\end{aligned}
$$

The fields are functions of $\sigma_{ \pm}=\tau \pm \sigma$, and summations over the indices $I \in\{1,2\}$ are implied. This action is in Lorentzian signature. We also have suppressed the indices $A$ displayed in [40], which run over the $N$ copies of the four-torus. Fermionic fields with and without a tilde correspond to the right and left-movers respectively.

The action is invariant up to boundary terms under the four supersymmetric transformations. These terms are given by

$$
\begin{array}{ll}
\delta_{1} S=\int d \tau \epsilon_{1}\left(\Psi^{1} \partial_{-} X^{2}-\Psi^{2 \dagger} \partial_{-} X^{1}\right), & \delta_{1^{\dagger}} S=\int d \tau \epsilon_{1 \dagger}\left(\Psi^{1 \dagger} \partial_{-} X^{2 \dagger}-\Psi^{2} \partial_{-} X^{1 \dagger}\right), \\
\delta_{2} S=\int d \tau \epsilon_{2}\left(\Psi^{2} \partial_{-} X^{2}-\Psi^{1 \dagger} \partial_{-} X^{1}\right), & \delta_{2^{\dagger}} S=\int d \tau \epsilon_{2 \dagger}\left(\Psi^{2 \dagger} \partial_{-} X^{2 \dagger}-\Psi^{1} \partial_{-} X^{1 \dagger}\right)
\end{array}
$$

where the transformations are generated by the four left-moving charges $G^{a}, G^{a \dagger}, a \in\{1,2\}$. The above expressions are evaluated at the boundary located at $\sigma=\sigma_{0}$.

The right-moving supercharges $\tilde{G}^{a}, \tilde{G}^{a \dagger}$ give rise to boundary terms that can be obtained from the above expressions exchanging + and - .

One then has to determine the boundary conditions satisfied by the fields at the interface. From energy conservation at the boundary it is natural to require that

$$
\left.\left(T\left(\sigma_{+}\right)-\tilde{T}\left(\sigma_{-}\right)\right)\right|_{\sigma_{0}}=0
$$

There are however various possible choices for the boundary conditions satisfied by the supercharges. For $\mathcal{N}=(4,4)$ supersymmetries, one can generalize the A- and B-type boundary conditions which are used in the $\mathcal{N}=(2,2)$ case. The boundary conditions satisfied by the R-symmetry current can then be deduced from those of the supercharges. 


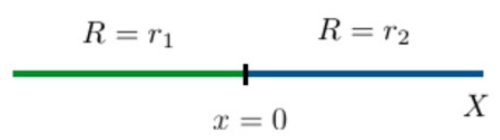

(a)

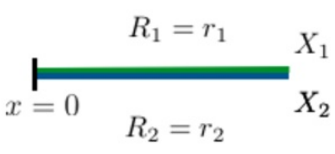

(b)

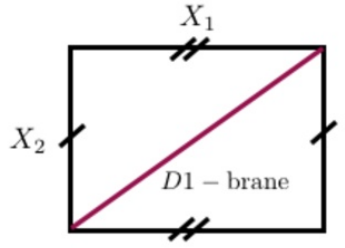

(c)

Figure 4. (a) An interface where radius of a compact boson jumps at $x=0$. (b) Folded boundary CFT and two bosons having different radiii. (c) Boundary conditions are equivalent to a diagonal D1 brane in a two dimensional torus.

Generally, if the interface preserves the global $\mathrm{SO}(4)$ symmetry, the supercharges have to satisfy

$$
\begin{aligned}
& \left(\begin{array}{c}
G^{1} \\
G^{2 \dagger}
\end{array}\right) \pm\left(\begin{array}{c}
\tilde{G}^{1} \\
\tilde{G}^{2 \dagger}
\end{array}\right)=0 \\
& \left(\begin{array}{c}
G^{1 \dagger} \\
G^{2}
\end{array}\right) \pm\left(\begin{array}{c}
\tilde{G}^{1 \dagger} \\
\tilde{G}^{2}
\end{array}\right)=0
\end{aligned}
$$

The analog of B-type boundary conditions is obtained by taking the minus sign in the above equations. In this case the $\mathrm{SU}(2)_{\mathrm{L}} \times \mathrm{SU}(2)_{\mathrm{R}}$ R-currents satisfy the boundary conditions

$$
J^{\alpha}\left(\sigma_{+}\right)-\tilde{J}^{\alpha}\left(\sigma_{-}\right)=0, \quad \alpha \in\{1,2,3\}
$$

Now we will examine super conformal field theories with two branches joining along the same boundary. For simplicity, we will consider only jumps in the radii of the target space torus across the interface. Using the folding trick as in [3] (see figure 4), we can show that this setup is equivalent to considering the direct product of two $\mathcal{N}=(4,4)$ super conformal field theories with a boundary.

In the free field limit, the boundary conditions satisfied by the bosons would again be given by $[3,30]$

$$
\frac{\dot{X}_{1}^{I}}{r_{1}^{I}}=\left.\frac{\dot{X}_{2}^{I}}{r_{2}^{I}}\right|_{\sigma=\sigma_{0}},\left.\quad \sum_{i=1,2} r_{i}^{I} X_{i}^{\prime I}\right|_{\sigma=\sigma_{0}}=0
$$

Picking the B-type conditions as discussed above, we have that an infinitesimal transformation generated by $G_{i}^{1}-\tilde{G}_{i}^{1}$ produces the following boundary terms in the action of the folded theory,

$$
\begin{aligned}
\delta_{1-\tilde{1}} S= & \sum_{i=1,2} \int d \tau \epsilon^{i}\left(r_{i}^{2}\left(\Psi_{i}^{1}-\tilde{\Psi}_{i}^{1}\right) \frac{\dot{X}_{i}^{2}}{r_{i}^{2}}-\frac{\left(\Psi_{i}^{1}+\tilde{\Psi}_{i}^{1}\right)}{r_{i}^{2}}\left(r_{i}^{2} X_{i}^{\prime 2}\right)\right. \\
& \left.-r_{i}^{1}\left(\Psi^{2 \dagger}-\tilde{\Psi}^{2 \dagger}\right) \frac{\dot{X}_{i}^{1}}{r_{i}^{1}}+\frac{\left(\Psi^{2 \dagger}+\tilde{\Psi}^{2 \dagger}\right)}{r_{1}^{1}} r_{1}^{1} X_{i}^{\prime 1}\right)
\end{aligned}
$$


where we have taken $\epsilon_{1}^{i}=-\epsilon_{\tilde{1}}^{i}=\epsilon^{i}$. The boundary terms would vanish upon imposing the boundary conditions (6.6) in case we have $\epsilon^{i}=\epsilon$ and

$$
\sum_{i=1,2} r_{i}^{J}\left(\Psi_{i}^{I}-\tilde{\Psi}_{i}^{I}\right)=0, \quad \frac{\Psi_{1}^{I}+\tilde{\Psi}_{1}^{I}}{r_{1}^{J}}=\frac{\Psi_{2}^{I}+\tilde{\Psi}_{2}^{I}}{r_{2}^{J}}
$$

Here, $J \neq I$. These conditions are precisely the fermionic analog of (6.6). We could repeat this procedure with the other three left- and right-moving pairs of supersymmetric transformations. Since the same fermionic fields $\Psi^{I}$ are transformed to different bosonic fields $X^{I}$, the radii $r_{i}^{I}$ of the bosonic fields $X_{i}^{I}$ in each branch are not independent for different $I$. The simplest choice that would ensure the preservation of one copy of the $\mathcal{N}=4$ supersymmetry would be setting all the radii at the different branches to the same value, i.e.

$$
r_{i}^{I}=r_{i}^{J}=r_{i}
$$

The conformal supercharges at each junction would then satisfy the boundary conditions

$$
\sum_{i=1,2} T_{i}-\tilde{T}_{i}=0, \quad \sum_{i=1,2} G_{i}^{a}-\tilde{G}_{i}^{a}=0
$$

Note that these boundary conditions are consistent with the structure of the supergravity solutions. Since the internal moduli of the four-torus are turned off, all the radii of the torus are treated in the same way and the jump in radius is the same for all of them. Furthermore, the metric ansatz for the BPS Janus solution has only a manifest $S^{2}$ factor and, consequently, only SU(2) isometry. Similarly, the boundary condition (6.5) preserves only a combination of the $\mathrm{SU}(2)_{L} \times \mathrm{SU}(2)_{R}$ superconformal R-symmetry.

\subsection{Calculation of boundary entropy on the CFT side}

As a starting point we consider the conformal field theory defined by a single boson on a circle of radius $R$. This CFT has central charge $c=1$. The interface is defined by a jump of the radius of the boson across $x=0$, i.e. the boson has radius $R=r_{-}$for $x<0$ and radius $R=r_{+}$for $x>0$. The folding trick relates this theory to a CFT of two bosons with radii $r_{ \pm}$defined on the half-space $x>0$. The boundary condition on the single boson is given by (6.6) setting $i=1,2$ and $I=1$,

$$
\left.\partial_{\tau}\left(\cos \theta X^{1}-\sin \theta X^{2}\right)\right|_{\sigma=0}=\left.\partial_{\sigma}\left(\sin \theta X^{1}+\sin \theta X^{2}\right)\right|_{\sigma=0}=0
$$

Hence, after folding, these conformal interface conditions imply boundary conditions which describe a $D 1$-brane on the diagonal of a rectangular two-torus with radii $r_{+}$and $r_{-}$. The boundary entropy of this boundary CFT is defined to be

$$
g=\langle 0 \mid B\rangle
$$

The $g$-factor was calculated in [35] and is given by the tension of the diagonal D1-brane on the torus

$$
g=\frac{1}{\sqrt{2}} \sqrt{\frac{r_{-}}{r_{+}}+\frac{r_{+}}{r_{-}}}
$$

The boundary entropy is then given by the logarithm of the $g$-factor.

For a tensor product of $n$ bosons with the same jump in radius, the $g$-factor is $g^{n}$ and hence the boundary entropy is given by $S=n \log g$. Since the $g$-factor is related to the tension of the $D$-brane, the result is also valid for superconformal field theories with fermions. 


\subsection{Boundary entropy for non-susy Janus}

As argued in [30], the sigma-model for the $D 1 / D 5$ CFT is associated with the string frame metric, taking the form

$$
\int d^{2} z e^{-\phi} G_{\mu \nu}^{s} \partial X^{\mu} \partial X^{\nu}
$$

It follows from the Einstein frame metric (4.1) that the string frame metric does not have any factor of $e^{\phi}$, hence one identifies the radius on the boundary with the asymptotic value of $e^{-\phi / 2}$. Therefore,

$$
\frac{r_{+}}{r_{-}}=\frac{\lim _{x \rightarrow \infty} e^{-\phi / 2}}{\lim _{x \rightarrow-\infty} e^{-\phi / 2}}=\left(\frac{1+\sqrt{2} \gamma}{1-\sqrt{2} \gamma}\right)^{\frac{1}{2 \sqrt{2}}}
$$

The $D 1 / D 5$ CFT has central charge $c=6 N_{1} N_{5}$ and thus $4 N_{1} \times N_{5}$ bosons. The solution treats all internal directions equally, so that all the bosons jump by the same amount. The CFT calculation therefore gives

$$
S_{\text {bdy }}=4 N_{1} N_{5} \log \left(\frac{1}{\sqrt{2}} \sqrt{\left(\frac{1+\sqrt{2} \gamma}{1-\sqrt{2} \gamma}\right)^{\frac{1}{2 \sqrt{2}}}+\left(\frac{1-\sqrt{2} \gamma}{1+\sqrt{2} \gamma}\right)^{\frac{1}{2 \sqrt{2}}}}\right)
$$

Note that (6.16) agrees with the holographic result (4.12) obtained from the non-BPS Janus solution up to quadratic order in an expansion around $\gamma=0$. However, the agreement does not hold for higher-order terms.

\subsection{Boundary entropy for BPS Janus}

As it was argued in section 6.1 , the supersymmetric boundary conditions imply a jump of all scalars by the same amount. In this section we repeat the CFT calculation of the boundary entropy using the value of the radius jumps of the BPS Janus solution (5.1). Interestingly, we find complete agreement between the CFT calculation and the holographic calculation. Transforming the Einstein frame metric (5.1) to the string frame metric provides an expression for the radii $r_{ \pm}$of the compact bosons

$$
r_{ \pm}=\lim _{x \rightarrow \pm \infty}\left(f_{3}^{2} e^{-\phi / 2}\right)^{\frac{1}{2}}
$$

The relevant supergravity solution is the one where $\theta=0$, i.e. there is no jump in the mode dual to the $Z_{2}$ orbifold twist operator. In this case, one finds

$$
\frac{r_{+}}{r_{-}}=e^{\psi}
$$

and the boundary entropy for a single boson (6.13) becomes

$$
g=\sqrt{\cosh \psi}
$$

Hence, the boundary entropy for the $D 1 / D 5$ CFT becomes

$$
S_{\text {bdy }}=4 N_{1} N_{5} \log (g)=\frac{2}{3} c \log (g)=\frac{32 \pi^{3} L^{2}}{k_{10}^{2}} \cosh ^{2} \psi \log \cosh (\psi)
$$

where we used the formula (5.8) for the central charge. This result agrees exactly with the holographic result obtained in equation (5.23). 


\subsection{Deformation by the $Z_{2}$ orbifold twist operator}

It is known that the radius deformation of a compact boson is an exactly marginal deformation of the bulk theory, since the operator

$$
\delta S=\lambda \int d^{2} z \partial X \bar{\partial} X
$$

is a $\mathrm{U}(1) \times \mathrm{U}(1)$ current-current deformation [36]. The case of an interface located at $x=0$ is more complicated since the deformation is now not uniform,

$$
\delta S=\lambda \int d^{2} z(\theta(x) \partial X \bar{\partial} X-\theta(-x) \partial X \bar{\partial} X)
$$

where $\theta(x)$ is the step function. However, it has been shown in [37] that even in the presence of an interface, the perturbation is exactly marginal and modifies the gluing conditions according to (6.11). It seems likely that this statement can also be directly proven in conformal perturbation theory.

The general holographic result for the boundary entropy contains two deformation parameters, $\theta$ and $\psi$ respectively

$$
\begin{aligned}
S_{\mathrm{bdy}} & =\frac{32 \pi^{3} L^{2}}{k_{10}^{2}} \cosh ^{2} \psi \cosh ^{2} \theta \log (\cosh \theta \cosh \psi) \\
& =2 N_{\mathrm{D} 1} N_{\mathrm{D} 5} \log (\cosh \theta \cosh \psi)
\end{aligned}
$$

In the previous section we have set $\theta$ to zero. The deformation parameter $\theta$ corresponds to the $Z_{2}$ orbifold twist operator. It is intriguing that the parameter controlling the radius deformation and the $Z_{2}$ orbifold twist deformation appear in a completely symmetric way in the expression (6.23).

It is tempting to conjecture that (6.23) is the exact result of a CFT computation including both radius and orbifold deformations.

The orbifold deformation is obtained by perturbing the theory by a dimension $(h, \bar{h})=$ $(1,1)$ operator $\mathcal{T}_{0}$. The operator $\mathcal{T}_{0}$ can be obtained from the following operator product expansion,

$$
\begin{aligned}
\mathcal{T}_{0}(z, \bar{z})= & \frac{1}{2 \sqrt{2}}\left(\epsilon_{a b} \oint d w G^{+a}(w) \oint d \bar{w} \bar{G}^{+b}(\bar{w}) \Sigma^{(1 / 2,1 / 2)}(z, \bar{z})\right. \\
& \left.+\epsilon_{a b} \oint d w G^{-a}(w) \oint d \bar{w} \bar{G}^{-b}(\bar{w}) \bar{\Sigma}^{(1 / 2,1 / 2)}(z, \bar{z})\right)
\end{aligned}
$$

Here $G^{a b}(z)$ is the superconformal generator and $a$ labels the $\mathrm{SU}(2)_{R}$ charge, whereas $b$ labels the $\mathrm{SU}(2)_{I}$ charge. Hence, the jump of the modulus associated with the $Z_{2}$ orbifold twist operator corresponds to a deformation of the CFT by

$$
\delta S=\lambda \int d^{2} z\left(\theta(x) \mathcal{T}_{0}(z, \bar{z})-\theta(-x) \mathcal{T}_{0}(z, \bar{z})\right)
$$

A first consequence of the result (6.23) is that under an orbifold deformation, the first non-trivial change in the boundary entropy occurs at second order in $\theta$ and is given by 
setting $\psi=0$ and expanding

$$
S_{\mathrm{bdy}}=\frac{c}{6}\left(\theta^{2}-\frac{1}{6} \theta^{4}+O\left(\theta^{6}\right)\right)
$$

The fact that the term linear in $\theta$ vanishes is in agreement with the result obtained by conformal perturbation theory in [38]. As discussed in [38], the change of the $g$-function is given by

$$
\frac{\delta g}{g}=-\frac{\pi}{2} \theta A_{o r b}+O\left(\theta^{2}\right)
$$

Where $A_{\text {orb }}$ is the one-point function of the (unintegrated) operator in (6.25). Since the onepoint function of a single twist operator evaluated in the unperturbed bulk theory vanishes, the lowest nontrivial contribution could appear at second order, in agreement with (6.26).

Furthermore in appendix B we show that the operator product expansion of two twist fields is given by

$$
\lim _{|z-w| \rightarrow 0} \mathcal{T}_{0}(z, \bar{z}) \mathcal{T}_{0}(w, \bar{w})=\frac{1}{|z-w|^{4}}+\text { finite }
$$

Where the singular OPE is exactly of the same form as the OPE of the $\partial X \bar{\partial} X$ operator in the radius deformation (6.21). The correlation functions on the plane which enter in the conformal perturbation theory are completely determined by the singular part of the OPE. Consequently, the radial perturbation (6.22) and the twist field perturbation (6.25) lead to the same change in the boundary entropy. Note however that due to the complexity of higher order conformal perturbation theory an explicit higher order calculation has not been performed.

Another view point of the twist mode deformation may be useful here. The orbifold point is after all one particular point in the full moduli space, and the twist modes of the orbifold CFT are only some of the many possible Kähler moduli deformations. To take a concrete example, consider the orbifold point $T^{4} / \mathbb{Z}_{2}$ of a $K_{3}$ surface. The number of distinct two forms is $b_{2}=22$ for a $K_{3}$ surface but the corresponding number on a $T^{4}$ is only 6 , which can roughly be identified with the world-sheet $B$-fields in the sigma model on $T^{4} / \mathbb{Z}_{2}$. The remaining 16 modes arise in the sigma-model precisely as twist-fields deformations. From this perspective then, while the twist-fields have a very different representation in the orbifold CFT, their underlying origin in the moduli space of $K_{3} 3$ surfaces are not so fundamentally different from the world-sheet $B$ fields. It is thus tempting to compare the supergravity result with a CFT computation of the boundary entropy in the presence of both $B$-field and radii jumps on a free CFT, where the jump of the $B$-field is taken to be proportional to that of the twists field couplings read off from the supergravity solution, i.e. we take $B^{ \pm}= \pm 4 n k \sinh \theta$ where $n$ is a proportionality constant to be determined. Our normalization is such that $2 B$ couples to the operator $\partial_{+} X^{1} \partial_{-} X^{2}-\partial_{+} X^{2} \partial_{-} X^{1}$. The computation is detailed in appendix $\mathrm{C}$. The result of the computation gives, remarkably for the naive choice of $n=\frac{1}{2}$, precisely $g=\cosh \theta \cosh \psi$, yielding complete agreement with the supergravity theory.

It would be very interesting to perform a direct calculation in the orbifold CFT, perhaps along the lines of [37] to show that (6.23) is indeed the boundary entropy of a deformed theory including the twist deformation. 


\section{Conclusions}

In this paper we have used the AdS/CFT correspondence to calculate holographically the entanglement entropy for an interface theory given by a marginal deformation of the $\left(T^{4}\right)^{N} / S_{N}$ orbifold CFT. In this theory, the volume of the target space four-torus and the mode dual to the $Z_{2}$ orbifold twist operator assume different values on each side of the interface. The calculation was performed using the half-BPS Janus solution obtained in $[24]$ as the gravitational dual for the interface theory.

We found exact agreement between holographic and CFT calculations in the case in which the jump in the moduli of the conformal field theory corresponds to a change of the six-dimensional dilaton in the supergravity solution. It appears that the boundary entropy is protected by supersymmetry, even though it is not an index.

The supergravity solution also permits the calculation of the entanglement entropy for a jump of the $Z_{2}$ orbifold twist operator of the $\left(T^{4}\right)^{N} / S_{N}$ orbifold CFT. It is interesting to note that the expression for the boundary entropy is completely symmetric in the jump parameters related to the volume and twist mode deformations.

Since the marginal operator associated with the twist mode deformation is a twist field (denoted with $\mathcal{T}_{0}$ ), there is no exact conformal field calculation to compare with the supergravity result. However, it is quite likely that the CFT calculation should agree with the supergravity computation. This observation leads to some interesting expectations regrading the properties of twist-field correlators. For a theory with a compact boson on the plane, the change of radius is related to a deformation by an operator of the form $J \bar{J}$, where $J$ is the R-symmetry current. This operator is exactly marginal [36]. Furthermore, correlation functions of an arbitrary number of $J$ operators are obtained from the two-point functions using Wick's theorem. We can consider a calculation of the boundary entropy using conformal perturbation theory along the lines of [38] for both the radius and the twist field deformations. The result of both calculations are expected to have exactly the same form. This observation suggests that the correlation functions for the $\mathcal{T}_{0}$ twist fields may be identical to the ones of the $J \bar{J}$ operators.

The study of interface conformal field theories has produced very exciting new developments with their application to the description of quantum wires (see e.g. [41-43]). Systems of quantum wires display a rich variety of interesting phenomena, with many different IR fixed points, whose physics is yet to be understood [44]. Networks of quantum wires can potentially be engineered using general multi-Janus solutions, and the entanglement entropy is only one of the many quantities which can be calculated using the AdS/CFT correspondence. It would be very interesting to obtain the boundary conditions at the interface and physical observables such as transport coefficient of the system, which can be extracted using bulk-boundary correlators in the Janus background.

In this paper, we have computed the entanglement entropy only for Janus solutions with $A d S_{3}$ asymptotics. However, the framework we have discussed at the beginning of this section can also be applied to BPS Janus solutions in different dimensions. In particular, it would be very interesting to obtain the entanglement entropy for the supersymmetric Janus solutions of [20] and [22, 45].

We plan to return to these interesting topics in the future. 


\section{Acknowledgments}

We are grateful to C. Bachas, E. D'Hoker, J. Estes, P. Kraus, D. Krym, S. Mathur and B. Shieh for useful conversations. The work of MG and MC was supported in part by NSF grant PHY-07-57702. The work of MC was supported in part by the 2009-10 Siegfried W. Ulmer Dissertation Year Fellowship of UCLA. The research of LYH at the Perimeter Institute is supported by the Government of Canada through Industry Canada and by the Province of Ontario through the Ministry of Research and Innovation.

\section{A BPS interface solution}

In this appendix we review the regular interface solution of type IIB supergravity which is locally asymptotic to $A d S_{3} \times S^{3} \times M_{4}$ and preserves eight of the sixteen supersymmetries of the AdS vacuum. More details can be found in [24] and [32].

\section{A.1 Local solutions}

The solutions are parameterized by two meromorphic functions $A(z), B(z)$ and two harmonic function $H(z, \bar{z}), K(z, \bar{z})$ (as well as their dual harmonic functions $\tilde{K}(z, \bar{z})$ ). All functions depend on the coordinates of the two-dimensional Riemann surface $\Sigma$ which has a boundary. The ten-dimensional metric is given by a fibration of $A d S_{2} \times S^{2} \times K_{3}$ over $\Sigma$.

$$
d s^{2}=f_{1}^{2} d s_{\mathrm{AdS}_{2}}^{2}+f_{2}^{2} d s_{\mathrm{S}^{2}}^{2}+f_{3}^{2} d s_{K_{3}}^{2}+\rho^{2} d z d \bar{z}
$$

The complex three-form is

$$
G=g_{a}^{(1)} f_{1}^{2} e^{a} \wedge \omega_{\mathrm{AdS}_{2}}+g_{a}^{(2)} f_{2}^{2} e^{a} \wedge \omega_{\mathrm{S}^{2}},
$$

The self-dual five-form flux is given by

$$
F_{5}=h_{a} f_{1}^{2} f_{2}^{3} e^{a} \wedge \omega_{\mathrm{AdS}_{2}} \wedge \omega_{\mathrm{S}^{2}},+\tilde{h}_{a} f_{3}^{4} e^{a} \wedge \omega_{K_{3}}
$$

Dilaton and axion are given by ${ }^{4}$

$$
\begin{aligned}
e^{-2 \phi} & =\frac{1}{4 K^{2}}\left((A+\bar{A}) K-(B+\bar{B})^{2}\right)\left((A+\bar{A}) K-(B-\bar{B})^{2}\right) \\
\chi & =\frac{i}{2 K}\left((A-\bar{A}) K-B^{2}+\bar{B}^{2}\right)
\end{aligned}
$$

The R-R four-form potential $C_{K}$ is given by

$$
C_{K}=-\frac{i}{2} \frac{B^{2}-\bar{B}^{2}}{A+\bar{A}}-\frac{1}{2} \tilde{K}
$$

and the metric factor of the compact four manifold $M_{4}$ is given by

$$
f_{3}^{4}=4 \frac{e^{-\phi} K}{A+\bar{A}}
$$

\footnotetext{
${ }^{4}$ In the previous paper we used $\phi$ which is related to the standard dilaton by $\phi=-2 \Phi$.
} 
The metric factors associated with $A d S_{2}$ and $S^{2}$ are given by

$$
\begin{aligned}
& f_{1}^{2}=\frac{e^{\phi}}{2 f_{3}^{2}} \frac{|H|}{K}\left((A+\bar{A}) K-(B-\bar{B})^{2}\right) \\
& f_{2}^{2}=\frac{e^{\phi}}{2 f_{3}^{2}} \frac{|H|}{K}\left((A+\bar{A}) K-(B+\bar{B})^{2}\right)
\end{aligned}
$$

The rank three anti-symmetric tensor fields can be expressed in terms of potentials

$$
\begin{aligned}
f_{1}^{2} \rho e^{\phi / 2} \operatorname{Re}\left(g^{(1)}\right)_{z} & =\partial_{w} b^{(1)} \\
f_{2}^{2} \rho e^{\phi / 2} \operatorname{Re}\left(g^{(2)}\right)_{z} & =\partial_{w} b^{(2)} \\
f_{1}^{2} \rho e^{-\phi / 2} \operatorname{Im}\left(g^{(1)}\right)_{z}+\chi f_{1}^{2} \rho e^{\phi / 2} \operatorname{Re}\left(g^{(1)}\right)_{z} & =\partial_{w} c^{(1)} \\
f_{2}^{2} \rho e^{-\phi / 2} \operatorname{Im}\left(g^{(2)}\right)_{z}+\chi f_{2}^{2} \rho e^{\phi / 2} \operatorname{Re}\left(g^{(2)}\right)_{z} & =\partial_{w} c^{(2)}
\end{aligned}
$$

The potentials $c^{(1,2)}$ and $b^{(1,2)}$ are expressed in terms of the meromorphic and harmonic functions as follows

$$
\begin{aligned}
b^{(1)} & =-\frac{H(B+\bar{B})}{(A+\bar{A}) K-(B+\bar{B})^{2}}-h_{1}, & & h_{1}=\frac{1}{2} \int \frac{\partial_{w} H}{B}+c . c . \\
b^{(2)} & =-i \frac{H(B-\bar{B})}{(A+\bar{A}) K-(B-\bar{B})^{2}}+\tilde{h}_{1}, & \tilde{h}_{1} & =\frac{1}{2 i} \int \frac{\partial_{w} H}{B}+c . c . \\
c^{(1)} & =-i \frac{H(A \bar{B}-\bar{A} B)}{(A+\bar{A}) K-(B+\bar{B})^{2}}+\tilde{h}_{2}, & \tilde{h}_{2} & =\frac{1}{2 i} \int \frac{A}{B} \partial_{w} H+c . c . \\
c^{(2)} & =-\frac{H(A \bar{B}+\bar{A} B)}{(A+\bar{A}) K-(B-\bar{B})^{2}}+h_{2}, & h_{2} & =\frac{1}{2} \int \frac{A}{B} \partial_{w} H+c . c .
\end{aligned}
$$

\section{A.2 Page charges}

In this section we review the expressions for the Page charges of the BPS interface. Further details can be found in [32]. The Page charges are conserved and localized as well as related to the quantized number of branes in a supergravity solution [39]. In type IIB the Page charges for $N S 5$ and $D 5$-branes are given by

$$
Q_{\mathrm{NS} 5}=\int_{\mathrm{M}_{3}} H_{3}, \quad Q_{\mathrm{D} 5}=\int_{\mathrm{M}_{3}}\left(\tilde{F}_{3}+\chi H_{3}\right)
$$

The final expressions for the five brane charges are given by

$$
\begin{aligned}
Q_{\mathrm{NS} 5} & =4 \pi\left(\int_{\mathcal{C}} d z \partial_{z} b^{(2)}+c . c\right) \\
Q_{\mathrm{D} 5} & =4 \pi\left(\int_{\mathcal{C}} d z \partial_{z} c^{(2)}+c . c\right)
\end{aligned}
$$

Where $\mathcal{C}$ is a contour in the Riemann surface $\Sigma$ which produces a three-sphere in the asymptotic region together with the fibered $S^{2}$. For the solutions in this paper, the Riemann surface $\Sigma$ is the half-plane and the contours providing homology three-spheres are the ones which enclose a pole of the harmonic function $H$. 
The Page charges for D1-branes and fundamental strings are given by

$$
\begin{aligned}
Q_{\mathrm{D} 1} & =-\int_{\mathrm{M}_{7}}\left(e^{\phi} * \tilde{F}_{3}-4 C_{4} \wedge H_{3}\right) \\
Q_{\mathrm{F} 1} & =-\int_{\mathrm{M}_{7}}\left(e^{-\phi} * H_{3}-\chi e^{\phi} * \tilde{F}_{3}+4 C_{4} \wedge d C_{2}\right)
\end{aligned}
$$

The expressions for the one-brane charges (A.20) are more complicated due to the Hodge dual in their definition and the presence of Chern-Simons terms. The seven-manifold appearing in the above expressions is a product of $M_{4}$ and a homology three-sphere obtained from a contour $\mathcal{C}$ just as in the case of the five brane charges.

The D1-brane charge is given by

$$
\begin{aligned}
Q_{\mathrm{D} 1}= & 4 \pi\left\{\int_{\mathcal{C}} \frac{4 K}{A+\bar{A}} \frac{(A+\bar{A}) K-(B+\bar{B})^{2}}{(A+\bar{A}) K-(B-\bar{B})^{2}} i\left(\partial_{z} c^{(1)}-\chi \partial_{z} b^{(1)}\right) d z\right. \\
& \left.-2 \int_{\mathcal{C}}\left(i \frac{B^{2}-\bar{B}^{2}}{A+\bar{A}}+\tilde{K}\right) \partial_{z} b^{(2)} d z\right\}+ \text { c.c. }
\end{aligned}
$$

The fundamental string charge is given by

$$
\begin{aligned}
Q_{\mathrm{F} 1}= & 4 \pi\left\{\int_{\mathcal{C}} \frac{\left((A+\bar{A}) K-(B+\bar{B})^{2}\right)^{2}}{K(A+\bar{A})} i \partial_{z} b^{(1)} d z+2\left(\frac{i\left(B^{2}-\bar{B}^{2}\right)}{A+\bar{A}}+\tilde{K}\right) \partial_{z} c^{(2)} d z\right. \\
& \left.-\int_{\mathcal{C}} \frac{4 K}{A+\bar{A}} \frac{(A+\bar{A}) K-(B+\bar{B})^{2}}{(A+\bar{A}) K-(B-\bar{B})^{2}} i \chi\left(\partial_{z} c^{(1)}-\chi \partial_{z} b^{(1)}\right) d z\right\}+ \text { c.c. }
\end{aligned}
$$

\section{A.3 BPS Janus solution}

The BPS Janus solution is defined on the strip with coordinates

$$
w=x+i y, \quad x \in[-\infty,+\infty], y \in[0, \pi]
$$

The meromorphic and harmonic functions are given by

$$
\begin{aligned}
H & =-i L \sinh (w+\psi)+c . c . \\
A & =i k^{2} \frac{\cosh \theta+\sinh \theta \cosh w}{\sinh w} \\
B & =i k \frac{\cosh (w+\psi)}{\cosh \psi \sinh w} \\
K & =i \frac{\cosh \theta-\sinh \theta \cosh w}{\sinh w}+c . c .
\end{aligned}
$$

One obtains the expressions of section 5 by plugging these functions into the formulae given in appendix A.1 .

\section{B Operator product of twist fields}

It was argued that for the twist operator $\mathcal{T}_{0}$ given in (6.24) the two terms containing $\Sigma^{(1 / 2,1 / 2)}$ and $\bar{\Sigma}^{(1 / 2,1 / 2)}$ are identical (see for example [46]). It is convenient to evaluate the 

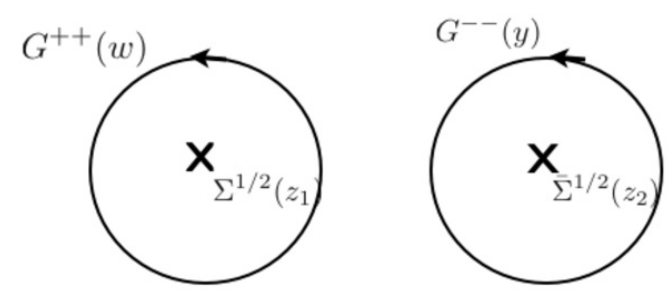

(a)

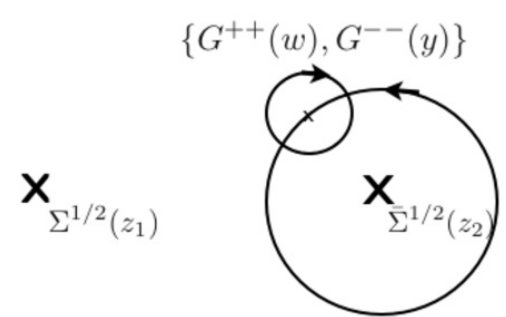

(b)

Figure 5. (a) Contour for holomorphic part before deformation (b) Contour deformation picks up anti-commutator of G's.

OPE as follows:

$$
\begin{aligned}
\lim _{z_{1} \rightarrow z_{2}} \mathcal{T}_{0}\left(z_{1}, \bar{z}_{1}\right) \mathcal{T}_{0}\left(z_{2}, \bar{z}_{2}\right)= & \frac{1}{2} \lim _{z_{1} \rightarrow z_{2}} \epsilon_{a b}\left(\oint_{C_{z_{1}}} d w G^{+a}(w) \oint_{C_{\bar{z}_{1}}} d \bar{w} \tilde{G}^{+b}(\bar{w})\right) \Sigma^{(1 / 2,1 / 2)}\left(z_{1}, \bar{z}_{1}\right) \\
& \epsilon_{c d}\left(\oint_{C_{z_{2}}} d y G^{-c}(y) \oint_{C_{\bar{z}_{2}}} d \bar{y} \tilde{G}^{-d}(\bar{y})\right) \bar{\Sigma}^{(1 / 2,1 / 2)}\left(z_{2}, \bar{z}_{2}\right)
\end{aligned}
$$

One can now deform the w-contour $C$ (see figure 5) which surrounds $z_{1}$ in such a way that the contour will surround $z_{2}$. Since $G^{+a}$ annihilates $\bar{\Sigma}$ the only relevant term comes from the OPE of two $G[47]$ :

$$
\begin{aligned}
& \oint_{C_{y}} d w G^{++}(w) G^{--}(y)=T(y)+\partial_{y} J^{3}(y) \\
& \oint_{C_{y}} d w G^{+-}(w) G^{-+}(y)=T(y)+\partial_{y} J^{3}(y)
\end{aligned}
$$

Which can be seen from the fact that the contour $C_{y}$ surrounds $y$ and in contour integration only the simple pole term in the GG OPE survives. Hence,

$$
\begin{aligned}
& \lim _{z_{1} \rightarrow z_{2}} \mathcal{T}_{0}\left(z_{1}, \bar{z}_{1}\right) \mathcal{T}_{0}\left(z_{2}, \bar{z}_{2}\right) \\
& \quad=\lim _{z_{1} \rightarrow z_{2}} \Sigma^{(1 / 2,1 / 2)}\left(z_{1}, \bar{z}_{1}\right) \oint_{C_{z_{2}}} d y\left(T(y)+\partial_{y} J^{3}(y)\right) \oint_{C_{z_{2}}} d \bar{y}\left(T(\bar{y})+\bar{\partial}_{y} J^{3}(\bar{y})\right) \bar{\Sigma}^{(1 / 2,1 / 2)}\left(z_{2}, \bar{z}_{2}\right)
\end{aligned}
$$

We use following OPE's

$$
\begin{aligned}
T(z) \Sigma^{1 / 2}(w) & =\frac{\partial_{w} \Sigma^{1 / 2}(w)}{z-w}+\frac{1}{2} \frac{\Sigma^{1 / 2}(w)}{(z-w)^{2}}+\cdots \\
J^{3}(z) \Sigma^{1 / 2}(w) & =\frac{1}{2} \frac{\Sigma^{1 / 2}(w)}{z-w}
\end{aligned}
$$


and hence,

$$
\begin{aligned}
\left(T(z)+\partial_{z} J^{3}(z)\right) \Sigma^{1 / 2}(w) & =\frac{\partial_{w} \Sigma^{1 / 2}(w)}{z-w}+\frac{1}{2} \frac{\Sigma^{1 / 2}(w)}{(z-w)^{2}}+\partial_{z} \frac{1}{2} \frac{\Sigma^{1 / 2}(w)}{z-w} \\
& =\frac{\partial_{w} \Sigma^{1 / 2}(w)}{z-w}
\end{aligned}
$$

The anti-holomorphic part leads to an analogous result. Hence the $\mathcal{T}_{0} \mathcal{T}_{0}$ OPE (B.3) becomes

$$
\begin{aligned}
\lim _{z_{1} \rightarrow z_{2}} \mathcal{T}_{0}\left(z_{1}, \bar{z}_{1}\right) \mathcal{T}_{0}\left(z_{2}, \bar{z}_{2}\right) & =\Sigma^{(1 / 2,1 / 2)}\left(z_{1}, \bar{z}_{1}\right) \partial_{z_{2}} \partial_{\bar{z}_{2}} \bar{\Sigma}^{(1 / 2,1 / 2)}\left(z_{2}, \bar{z}_{2}\right) \\
& =\partial_{z_{2}} \partial_{\bar{z}_{2}} \frac{1}{\left(z_{1}-z_{2}\right)\left(\bar{z}_{1}-\bar{z}_{2}\right)}+\cdots \\
& =\frac{1}{\left|z_{1}-z_{2}\right|^{4}}+\cdots
\end{aligned}
$$

Where the dots denote nonsingular terms as $z_{1} \rightarrow z_{2}$. We used the fact that the OPE of the chiral and antichiral twist field are given by

$$
\lim _{z_{1} \rightarrow z_{2}} \Sigma^{(1 / 2,1 / 2)} \bar{\Sigma}^{(1 / 2,1 / 2)}=\frac{1}{\left(z_{1}-z_{2}\right)\left(\bar{z}_{1}-\bar{z}_{2}\right)}+\cdots
$$

\section{Boundary entropy with both radii and $B$ field jumps}

Consider starting off with two compact bosons from each side of the interface, which is the smallest number of fields that can be coupled to an antisymmetric $B$-field.

The action before folding is

$$
\int_{\sigma>0} d \sigma d \tau\left(r_{i}^{2} \eta^{a b} \partial_{a} X^{i} \partial_{b} X^{i}+2 \epsilon^{a b} b \partial_{a} X^{1} \partial_{b} X^{2}\right)+\int_{\sigma<0} d \sigma d \tau\left(\tilde{r}_{i}^{2} \eta^{a b} \partial_{a} \tilde{X}^{i} \partial_{b} \tilde{X}^{i}+2 \epsilon^{a b} \tilde{b} \partial_{a} \tilde{X}^{1} \partial_{b} \tilde{X}^{2}\right)
$$

where $i \in\{1,2\}$, and $a, b \in\{\sigma, \tau\}$. After folding the theory in $\sigma<0$, by sending $\sigma \rightarrow-\sigma$, we obtain the same action except $\tilde{b}$ changes sign. The boundary conditions following from the vanishing of the boundary terms upon variation of the fields are given by

$$
\begin{aligned}
r_{i}^{2} \partial_{\sigma} X^{i}+\tilde{r}_{i}^{2} \partial_{\sigma} \tilde{X}^{i}+\epsilon_{i j}\left(b \partial_{\tau} X^{j}-\tilde{b} \partial_{\tau} \tilde{X}^{j}\right) & =0, & & i=1,2 \\
\partial_{\tau} X^{i} & =\partial_{\tau} \tilde{X}^{i}, & i & =1,2
\end{aligned}
$$

The boundary conditions (C.2) correspond to the open strings picture, where boundary conditions are imposed on the spatial boundary $\sigma=0$ of the world-sheet. By exchanging $\sigma \leftrightarrow \tau$ one obtains the closed string picture, where a closed string propagates and the boundary conditions are imposed at a fixed time $\tau=0$.

The mode expansion of the closed strings are given by (using the normalization in [35])

$$
X^{i}\left(\sigma_{\tau}\right)=x^{i}+2 \omega_{i} \sigma+\tau G^{i j}\left(p_{j}-2 B_{j k} \omega^{k}\right)+\frac{i}{2} \sum_{n \neq 0} \frac{1}{n}\left(\alpha_{L, n}^{i} e^{-2 i n \sigma_{+}}+\alpha_{R, n}^{i} e^{-2 i n \sigma_{-}}\right)
$$

where $p_{i}$ and $\omega^{i}$ are the momentum and winding respectively and in our normalization these are integers. The target space metric $G_{i j}$ is again simply $\delta_{i j} r_{i}^{2}$, whereas $B_{i j}=b$. We 
have similar expressions for the tilde variables, except the sign of $\tilde{b}$ is flipped. Note that the position of the indices are important.

Using the above expansion, the boundary conditions (C.2) implies here

$$
p_{i}=-\tilde{p}_{i}, \quad \omega^{i}=\tilde{\omega}^{i}
$$

These relations dictate how we build the corresponding boundary state $|B\rangle$. However, to extract the boundary entropy $\langle 0 \mid B\rangle$ we need only to know the zero modes contribution of $\langle B|\exp (-i \pi H / T)| B\rangle$, where $H$ is the closed string Hamiltonian and $T$ the periodicity in the time direction. The Hamiltonian is given by

$$
H=\frac{1}{4}\left(p_{\mu} G^{\mu \nu} p_{\nu}+4 \omega^{\mu}\left(G-B \cdot G^{-1} \cdot B\right)_{\mu \nu} \omega^{\nu}+4 \omega^{\mu}\left(B \cdot G^{-1}\right)_{\mu}^{\nu} p_{\nu}\right)+N_{L}+N_{R}
$$

where indices $\mu, \nu$ denote summation over both tilded and untilded fields. $N_{L, R}$ are the level of the left and right moving modes.

Using (C.4), we can rewrite the zero-mode contribution of the Hamiltonian as a matrix multiplication $P^{T} \cdot M \cdot P$, where

$$
P=\left(\begin{array}{c}
p_{1} \\
p_{2} \\
\omega_{1} \\
\omega_{2}
\end{array}\right), \quad M=\frac{1}{4}\left(\begin{array}{cccc}
M_{11} & 0 & 0 & M_{14} \\
0 & M_{22} & M_{23} & 0 \\
0 & M_{32} & M_{33} & 0 \\
M_{41} & 0 & 0 & M_{44}
\end{array}\right)
$$

and $M$ is a symmetric matrix where

$$
\begin{array}{ll}
M_{11}=\frac{1}{r_{1}^{2}}+\frac{1}{\tilde{r}_{1}^{2}}, & M_{22}=\frac{1}{r_{2}^{2}}+\frac{1}{\tilde{r}_{2}^{2}} \\
M_{33}=4\left(r_{1}^{2}+\frac{b^{2}}{r_{2}^{2}}+\tilde{r}_{1}^{2}+\frac{\tilde{b}^{2}}{\tilde{r}_{2}^{2}}\right), & M_{44}=4\left(r_{2}^{2}+\frac{b^{2}}{r_{1}^{2}}+\tilde{r}_{2}^{2}+\frac{\tilde{b}^{2}}{\tilde{r}_{1}^{2}}\right) \\
M_{14}=-2\left(\frac{b}{r_{1}^{2}}+\frac{\tilde{b}}{r_{1}^{2}}\right), & M_{23}=2\left(\frac{b}{r_{2}^{2}}+\frac{\tilde{b}}{r_{2}^{2}}\right)
\end{array}
$$

Th boundary entropy $g_{b}$ is then given by

$$
g_{b}=|\operatorname{det} M|^{1 / 4}=\left|\frac{\left((b-\tilde{b})^{2}+\left(r_{1}^{2}+\tilde{r}_{1}^{2}\right)\left(r_{2}^{2}+\tilde{r}_{2}^{2}\right)\right)^{2}}{16 r_{1}^{2} r_{2}^{2} \tilde{r}_{1}^{2} \tilde{r}_{2}^{2}}\right|^{1 / 4}
$$

Now using (5.10), (5.9), we take

$$
b=-\tilde{b}=4 n k \sinh \theta, \quad r_{i}=\sqrt{\frac{2 k e^{\psi}}{\cosh \psi}}, \quad \tilde{r}_{i}=\sqrt{\frac{2 k e^{-\psi}}{\cosh \psi}}
$$

For $n=1 / 2$

$$
g_{b}=\cosh \theta \cosh \psi
$$

Since we are considering two directions along $T^{4}$ at the same time in the above calculation, the final result, including the other two orthogonal directions in $T^{4}$ and all the copies in the symmetric product, is indeed

$$
S_{\mathrm{bdy}}=2 N_{\mathrm{D} 1} N_{\mathrm{D} 5} \log (\cosh \theta \cosh \psi)
$$

in complete agreement with the supergravity result (6.23). 
Open Access. This article is distributed under the terms of the Creative Commons Attribution Noncommercial License which permits any noncommercial use, distribution, and reproduction in any medium, provided the original author(s) and source are credited.

\section{References}

[1] J.L. Cardy, Boundary Conditions, Fusion Rules and the Verlinde Formula, Nucl. Phys. B 324 (1989) 581 [SPIRES].

[2] M. Oshikawa and I. Affleck, Boundary conformal field theory approach to the critical two-dimensional Ising model with a defect line, Nucl. Phys. B 495 (1997) 533 [cond-mat/9612187] [SPIRES].

[3] C. Bachas, J. de Boer, R. Dijkgraaf and H. Ooguri, Permeable conformal walls and holography, JHEP 06 (2002) 027 [hep-th/0111210] [SPIRES].

[4] I. Affleck and A.W.W. Ludwig, Universal noninteger 'ground state degeneracy' in critical quantum systems, Phys. Rev. Lett. 67 (1991) 161 [SPIRES].

[5] C.L. Seaman et al., Evidence for non-Fermi liquid behavior in the Kondo alloy $Y_{1-x} U x P d_{3}$, Phys. Rev. Lett. 67 (1991) 2882.

[6] P. Calabrese and J.L. Cardy, Entanglement entropy and quantum field theory, J. Stat. Mech. (2004) P06002 [hep-th/0405152] [SPIRES].

[7] J.M. Maldacena, The large- $N$ limit of superconformal field theories and supergravity, Adv. Theor. Math. Phys. 2 (1998) 231 [Int. J. Theor. Phys. 38 (1999) 1113] [hep-th/9711200] [SPIRES].

[8] S.S. Gubser, I.R. Klebanov and A.M. Polyakov, Gauge theory correlators from non-critical string theory, Phys. Lett. B 428 (1998) 105 [hep-th/9802109] [SPIRES].

[9] E. Witten, Anti-de Sitter space and holography, Adv. Theor. Math. Phys. 2 (1998) 253 [hep-th/9802150] [SPIRES].

[10] A. Karch and L. Randall, Open and closed string interpretation of SUSY CFT's on branes with boundaries, JHEP 06 (2001) 063 [hep-th/0105132] [SPIRES].

[11] O. Aharony, O. DeWolfe, D.Z. Freedman and A. Karch, Defect conformal field theory and locally localized gravity, JHEP 07 (2003) 030 [hep-th/0303249] [SPIRES].

[12] D. Bak, M. Gutperle and S. Hirano, A dilatonic deformation of $A d S_{5}$ and its field theory dual, JHEP 05 (2003) 072 [hep-th/0304129] [SPIRES].

[13] A. Clark and A. Karch, Super Janus, JHEP 10 (2005) 094 [hep-th/0506265] [SPIRES].

[14] O. Lunin, On gravitational description of Wilson lines, JHEP 06 (2006) 026 [hep-th/0604133] [SPIRES].

[15] O. Lunin, 1/2-BPS states in M-theory and defects in the dual CFTs, JHEP 10 (2007) 014 [arXiv: 0704.3442] [SPIRES].

[16] S. Yamaguchi, Bubbling geometries for half BPS Wilson lines, Int. J. Mod. Phys. A 22 (2007) 1353 [hep-th/0601089] [SPIRES].

[17] J. Gomis and F. Passerini, Holographic Wilson loops, JHEP 08 (2006) 074 [hep-th/0604007] [SPIRES]. 
[18] J. Gomis and C. Romelsberger, Bubbling defect CFT's, JHEP 08 (2006) 050 [hep-th/0604155] [SPIRES].

[19] E. D'Hoker, J. Estes and M. Gutperle, Exact half-BPS Type IIB interface solutions I: Local solution and supersymmetric Janus, JHEP 06 (2007) 021 [arXiv:0705.0022] [SPIRES].

[20] E. D'Hoker, J. Estes and M. Gutperle, Exact half-BPS Type IIB interface solutions II: Flux solutions and multi-Janus, JHEP 06 (2007) 022 [arXiv:0705.0024] [SPIRES].

[21] E. D'Hoker, J. Estes, M. Gutperle and D. Krym, Exact Half-BPS Flux Solutions in M-theory I, Local Solutions, JHEP 08 (2008) 028 [arXiv:0806.0605] [SPIRES].

[22] E. D'Hoker, J. Estes, M. Gutperle and D. Krym, Exact Half-BPS Flux Solutions in M-theory II: Global solutions asymptotic to $A d S_{7} \times S^{4}$, JHEP 12 (2008) 044 [arXiv:0810.4647] [SPIRES].

[23] E. D'Hoker, J. Estes, M. Gutperle and D. Krym, Exact Half-BPS Flux Solutions in M-theory III: Existence and rigidity of global solutions asymptotic to AdS4 $\times S 7$, JHEP 09 (2009) 067 [arXiv:0906.0596] [SPIRES].

[24] M. Chiodaroli, M. Gutperle and D. Krym, Half-BPS Solutions locally asymptotic to $A d S_{3} \times S^{3}$ and interface conformal field theories, JHEP 02 (2010) 066 [arXiv:0910.0466] [SPIRES].

[25] J. Kumar and A. Rajaraman, New supergravity solutions for branes in $A d S_{3} \times S^{3}$, Phys. Rev. D 67 (2003) 125005 [hep-th/0212145] [SPIRES].

[26] J. Kumar and A. Rajaraman, Supergravity solutions for $A d S_{3} \times S^{3}$ branes, Phys. Rev. D 69 (2004) 105023 [hep-th/0310056] [SPIRES].

[27] J. Kumar and A. Rajaraman, Revisiting D-branes in $A d S_{3} \times S^{3}$, Phys. Rev. D 70 (2004) 105002 [hep-th/0405024] [SPIRES].

[28] S. Ryu and T. Takayanagi, Holographic derivation of entanglement entropy from AdS/CFT, Phys. Rev. Lett. 96 (2006) 181602 [hep-th/0603001] [SPIRES].

[29] S. Ryu and T. Takayanagi, Aspects of holographic entanglement entropy, JHEP 08 (2006) 045 [hep-th/0605073] [SPIRES].

[30] T. Azeyanagi, A. Karch, T. Takayanagi and E.G. Thompson, Holographic Calculation of Boundary Entropy, JHEP 03 (2008) 054 [arXiv: 0712.1850] [SPIRES].

[31] D. Bak, M. Gutperle and S. Hirano, Three dimensional Janus and time-dependent black holes, JHEP 02 (2007) 068 [hep-th/0701108] [SPIRES].

[32] M. Chiodaroli, M. Gutperle. L-Y. Hung and D. Krym, String Junctions and Holographic Interface Solutions in $A d S_{3} \times S_{3} \times K_{3}$, to appear.

[33] I. Papadimitriou and K. Skenderis, Correlation functions in holographic RG flows, JHEP 10 (2004) 075 [hep-th/0407071] [SPIRES].

[34] J.D. Brown and M. Henneaux, Central Charges in the Canonical Realization of Asymptotic Symmetries: An Example from Three-Dimensional Gravity, Commun. Math. Phys. 104 (1986) 207 [SPIRES].

[35] S. Elitzur, E. Rabinovici and G. Sarkissian, On least action D-branes, Nucl. Phys. B 541 (1999) 246 [hep-th/9807161] [SPIRES]. 
[36] S. Chaudhuri and J.A. Schwartz, A criterion for integrably marginal operators, Phys. Lett. B 219 (1989) 291 [SPIRES].

[37] S. Fredenhagen, M.R. Gaberdiel and C.A. Keller, Symmetries of perturbed conformal field theories, J. Phys. A 40 (2007) 13685 [arXiv:0707.2511] [SPIRES].

[38] D.R. Green, M. Mulligan and D. Starr, Boundary Entropy Can Increase Under Bulk RG Flow, Nucl. Phys. B 798 (2008) 491 [arXiv:0710.4348] [SPIRES].

[39] D. Marolf, Chern-Simons terms and the three notions of charge, hep-th/0006117 [SPIRES].

[40] J.R. David, G. Mandal and S.R. Wadia, Microscopic formulation of black holes in string theory, Phys. Rept. 369 (2002) 549 [hep-th/0203048] [SPIRES].

[41] C.L. Kane and M.P.A. Fisher, Transmission through barriers and resonant tunneling in an interacting one-dimensional electron gas, Phys. Rev. B 46 (1992) 15233 [SPIRES].

[42] E. Wong and I. Affleck, Tunneling in quantum wires: A Boundary conformal field theory approach, Nucl. Phys. B 417 (1994) 403 [SPIRES].

[43] B. Bellazzini, M. Burrello, M. Mintchev and P. Sorba, Quantum Field Theory on Star Graphs, arXiv:0801.2852 [SPIRES].

[44] M. Oshikawa, C. Chamon and I. Affleck, Junctions of three quantum wires, J. Stat. Mech. (2006) P02008 [cond-mat/0509675] [SPIRES].

[45] E. D'Hoker, J. Estes, M. Gutperle and D. Krym, Janus solutions in M-theory, JHEP 06 (2009) 018 [arXiv: 0904.3313] [SPIRES].

[46] E. Gava and K.S. Narain, Proving the pp-wave / CFT(2) duality, JHEP 12 (2002) 023 [hep-th/0208081] [SPIRES].

[47] M. Yu, The unitary representations of the $N=4 \mathrm{SU}(2)$ extended superconformal algebras, Nucl. Phys. B 294 (1987) 890 [SPIRES]. 